Original Contribution

\title{
DYNAMIC FOLLOW-UP AND PROVING THROUGH COMPUTED TOMOGRAPHY AND MAGNETIC RESONANCE IMAGING OF CHANGES IN SECONDARY MENINGOENCEPHALITIS FROM SUPPURATIVE LEFT MAXILLARY SINUSITIS
}

\author{
M. Mitev ${ }^{*}$, L. Pekova ${ }^{2}$, St. Valkanov ${ }^{3}$ \\ ${ }^{1}$ Department of Medical Physics, Biophysics, Roentgenology and Radiology, Department of \\ Diagnostic Imaging, University Hospital "St. Kirkovich", Medical Faculty, Trakia University, Stara \\ Zagora, Bulgaria \\ ${ }^{2}$ Department of Infectious Diseases, Clinic of Infectious Diseases, University Hospital, \\ Medical Faculty, Trakia University, Stara Zagora, Bulgaria \\ ${ }^{3}$ Department of Surgery, Neurosurgery and Urology and Anesthesiology, Clinic of Neurosurgery, \\ Medical Faculty, Trakia University, Stara Zagora, Bulgaria
}

\begin{abstract}
Infections of the nervous system and its belonging structures have often been reported as life-threatening conditions. Purpose: the results of dynamic follow-up and proving of changes in secondary meningoencephalitis from suppurative left maxillary sinusitis are presented using the imaging methods CT and MRI. Methods: diagnostic imaging (CT, MRI), of acute disease in a 13-year-old child with progressive development and proving of secondary bacterial meningoencephalitis. Result: the control CT after two days showed dynamics in the imaging CT finding with the appearance of changes in the type of brain oedema and development of pansinusitis. The MRI data described corresponded to changes as at meningoencephalitis in progress.Conclusion: The extension of the diagnostic algorithm with MRI, with the administration of intravenous contrast medium, clearly demonstrated changes characteristic of meningoencephalitis.
\end{abstract}

Key words: CT, MRT, secondary meningoencephalitis, pediatric case

\begin{abstract}
INTRODUCTION
Infections of the nervous system and its belonging structures have often been reported as life-threatening conditions. According to several studies and cases in diagnostic practice, the prognosis for the outcome of the disease depends mainly on the rapid identification of the site of inflammation and the pathogen to initiate effective antimicrobial treatment as early as possible (1). Meningitis is the most common intracranial complication of sinusitis and serious infection, often with complications and a serious threat to patients (2). Acute
\end{abstract}

\footnotetext{
Correspondence to:D-r Mitko Atanasov Mitev, DM, Department of Medical Physics,

Biophysics, Roentgenology and Radiology, Faculty of Medicine, Department of Diagnostic Imaging, University Hospital "St. Kirkovich", Trakia University - Stara Zagora, 6000 Stara Zagora, Bulgaria, Mobile: +359 887 706079, E-mail: mitev.mitko69@gmail.com
}

infectious diseases of the central nervous system, in particular, bacterial meningitis, are referred to as neurological emergencies, which often have no definitive symptoms or signs, complicating the diagnosis, and delayed diagnosis or treatment of bacterial meningitis and encephalitis leads to poor prognosis (4).

\section{PURPOSE}

The results of dynamic follow-up and proving of changes in secondary meningoencephalitis from suppurative left maxillary sinusitis are presented using the imaging methods - CT and MRI.

\section{MATERIAL AND METHODS}

This is a case of follow-up using diagnostic imaging (CT, MRI), of acute disease in a 13year-old child with progressive development and proving of secondary bacterial 
meningoencephalitis and subdural abscess, with an unspecified bacterial agent, but most likely to be concomitant of primary suppurative maxillary sinusitis caused by a highly virulent bacterial agent in combination with reduced immune status of the body. The CT study was performed on 64 MDCT "Siemens Definition AS". Scanning was performed with post-processing and was done at $0.6 \mathrm{~mm}$. MDVB was performed based on the obtained reconstructed images. The additional processing was executed with a protocol for virtual bronchoscopy. "Siemens" workstation was used with "Singovia VB20" software, and "Siemens Definition AS" workstations with the capacity to track and match the images in the axial, coronary, sagittal planes. Multiplanar reconstructions were performed by applying MIP techniques and capabilities to archive and export images and video. The optimized standard protocol was used (current strength 80-100 mAs, voltage $100 \mathrm{kV}, 3 \mathrm{~mm}$ beam collimation, reconstruction of $0.6 \mathrm{~mm}$, rotation speed at $0.5 \mathrm{sec}$, pitch D-FOV Large). Magnetic resonance imaging (MRI), native and with the administration of intravenous contrast medium (Clariscan, $20 \mathrm{ml}$ ), with a Siemens Magnetom Essenza 1.5 tesla was used. The data were processed on a Syngo.via VB20 workstation. The study was performed in the axial, coronary and sagittal planes. The pulse sequences are SE, RSE, DIX, VIBE fs. Images in $\mathrm{T} 1 \mathrm{w}$ and $\mathrm{T} 2 \mathrm{w}$ sequences were obtained.

\section{RESULTS}

A 13-year-old male child was examined. The patient was admitted to the hospital because of a disease with an acute onset, manifesting with a fever reaching up to 40.2 degrees $\mathrm{C}$, with shaking, muscle soreness throughout the body, severe headache in the left half of the head and the left eye. Parents also reported repeated vomiting without relief. Upon admission to the hospital, the patient was intoxicated and somnolent. The admitting physician described from the examination the throat redness, single superficial lymph nodes (LN) in the cervical area, measuring as much as pea grains, and the redness of the left upper eyelid. A progressive leukocytosis from 15 to 22.7 and an increase in CRP (C-reactive protein) of up to 40 units were found in the paraclinical analyses. Immediately after admission, a lumbar puncture was performed, the results of which indicated evidence of a purulent inflammatory process. His condition was getting worse; the magnetic resonance spectroscopy was becoming more demonstrative. Headache in the left half predominated, the swelling of the left eyelid increased. After puncture to the left eye, Staphylococcus epidermidis was isolated. No bacterial flora was isolated from aerobic hemoculture after seven days of cultivation. On day 3, the patient's condition worsens sharply, severe pain in the right leg occurred until it became impossible to move independently - in the upright position, he folded both legs, more the right one, which he pulled on stepping forward. Active movements were performed in a limited volume for the right limbs, for which reduced muscle strength and tone were found.

From the imaging studies performed on the patient's admission, from the CT scan of the head, no pathological changes in the area of the cranial bones, meninges, brain parenchyma, basal cisterns, and ventricular system were detected. Bilaterally, the sulci and gyri were well pronounced and of normal width. As a pathological finding, the filling of half of the left maxillary sinus with the fluid collection, with a density of $21 \mathrm{HE}$ (Hounsfield units), was defined, as well as areas of irregular thickening of the mucous membrane at the base of the right maxillary sinus and in the ethmoidal cells. On the left, parasagittally and frontally, a small hypodense area with adipose-equivalent density, with axial dimensions of $4 / 5 \mathrm{~mm}$ and a density of minus $90 \mathrm{HE}$ was seen. In conclusion, the changes described in the left maxillary sinus corresponded to purulent left maxillary sinusitis, chronic etmoiditis and right maxillary sinusitis. A small lipoma on the left frontally and parasagittally was also found (Figure 1-2).

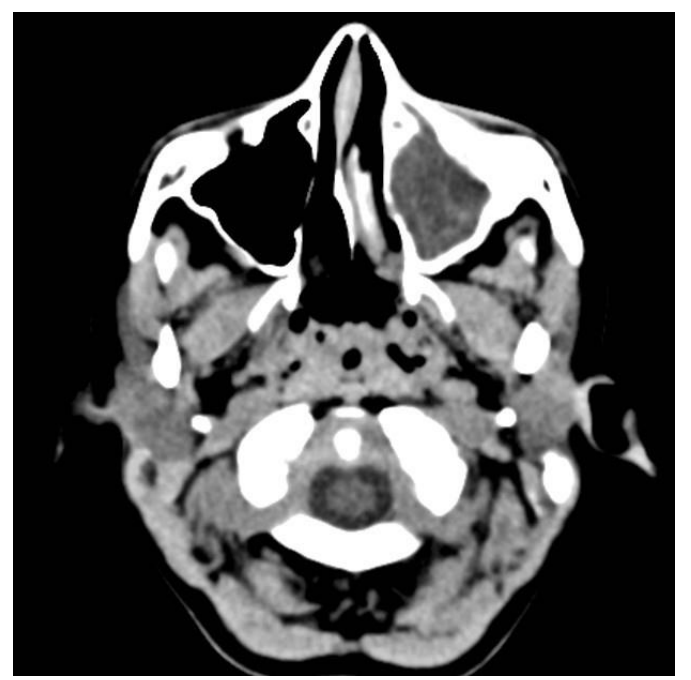

Figure 1. CT axial projection. Suppurative left maxillary sinusitis 


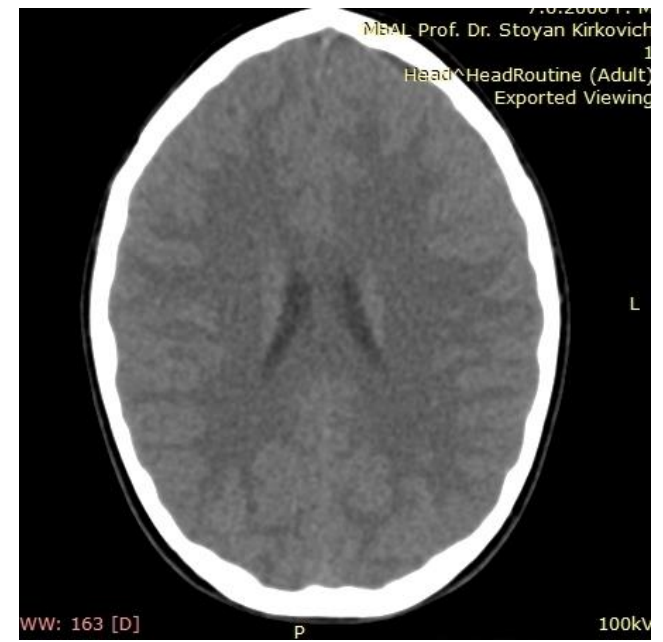

Figure 2. CT axial projection. A normal finding of the brain parenchyma and meninges

Due to the deterioration of the patient's condition after two days, in a control computer-tomographic study, dynamic changes were detected in the CT finding, in comparison with the previous study. The subarachnoid spaces along the convexity of the two upper cerebral hemispheres were narrowed, with changes more pronounced on the left frontotemporoparietally. Infratentorially and supratentorially, no focal changes in the brain parenchyma were detected. Liquid-equivalent collections appeared in the other paranasal cavities with a density of $20 \mathrm{HE}$. The swelling in the left upper eyelid increased (Figure 3).

The conclusion was reached for: cerebral oedema; suppurative left maxillary sinusitis; ethmoidal and maxillary exacerbated chronic sinusitis; some small lipoma on the left, frontally and parasagittally.

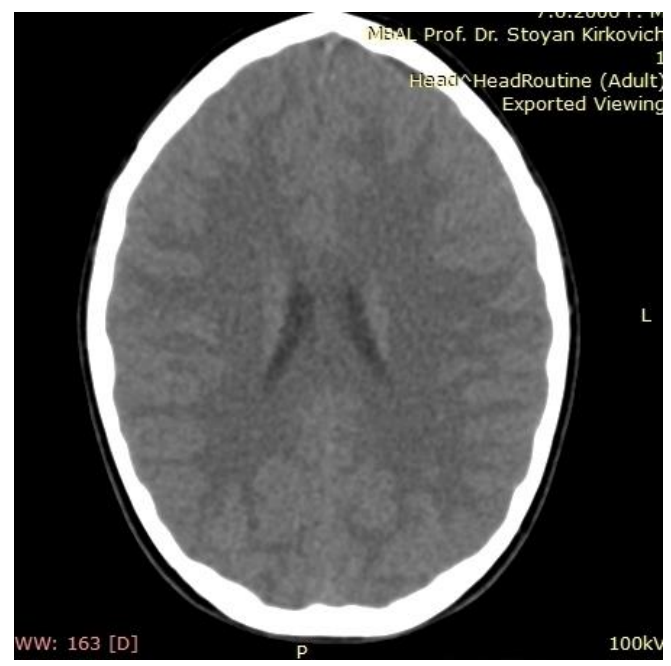

Figure 3. CT axial projection. Narrow subarachnoid spaces, bilateral, without focal changes
MITEV M., et al.

The diagnostic algorithm was extended by conducting a native MRI study, which revealed the following changes: the subarachnoid spaces along the convexity of the two upper cerebral hemispheres were narrowed. Homogeneous high-signal changes in $\mathrm{T} 2 \mathrm{w}$ sequences and low-signal ones in $\mathrm{T} 1 \mathrm{w}$ sequences were seen in the course of the meninges and the cortex parenchyma, bilaterally, frontally and on the left frontotemporoparietally. On the left, parietally and subdurally, there was a small welldelimited collection, high-signal in $\mathrm{T} 2 \mathrm{w}$ sequences and low-signal in $\mathrm{T} 1 \mathrm{w}$ and $\mathrm{T} 2$ FLAIR (fluid-attenuated inversion recovery). Brain ventricles and basal cisterns were of normal size, location, and signal intensity. The interhemispheric fissure is located in the middle. Supratentorial finding - on the left, frontally and parasagittally a low-signal sharply delimited lesion was seen with axial dimensions of $4 / 5 \mathrm{~mm}$, with the characteristic of adipose tissue known from the previous CT studies. Sellar and parasellar structures are normal. Retrobulbar spaces are normal. Infratentorially the cerebral parenchyma is free of pathological signal changes. Paranasal cavities in the study volume - high signal changes in the $\mathrm{T} 2 \mathrm{w}$ sequence in the half of the left maxillary sinus and low signal in the $\mathrm{T} 1 \mathrm{w}$ and T2 FLAIR. High signal changes in the base of the right maxillary sinus and the ethmoidal cells (Figure 4-5).

Conclusion: The MRI results described are most relevant to meningoencephalitis; cerebral oedema; lipoma on the left, frontally and parasagittally; suppurative left maxillary, ethmoidal sinusitis; small liquid collection on the left, subdurally.

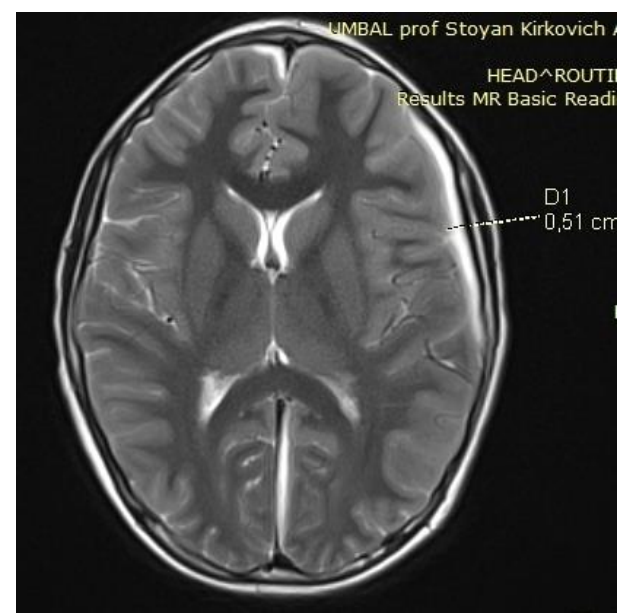

Figure 4. MRI T1 sequence, axial section. Low signal changes to the left, subdural and bilateral, frontal 


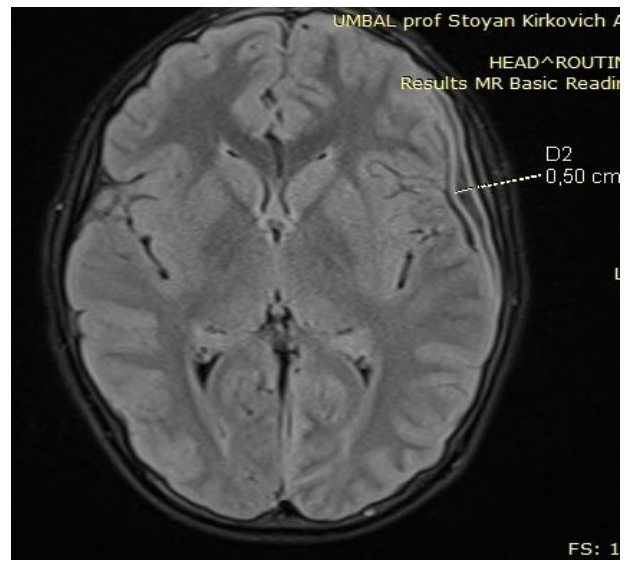

Figure 5. MRI, T2 sequence, axial section, high signal changes to the left, a subdural and bilateral frontal with dislocation of the interhemispheric furrow and compression of the lateral ventricle

On a control, CT study, at day 14 of the admission, a more limited fluid-equivalent convexity collection was found and localized on the left frontoparietally and subdurally, with axial dimensions reaching 76/ $16 \mathrm{~mm}$ and a finding density of $21 \mathrm{HE}$. Compression and dislocation of the surrounding parenchymal structures and complete smoothing and deletion of the proximal sulci and gyri were found. Presence of compression, slight stenosis and dislocation of the body of the ipsilateral lateral ventricle. Displacement of the interhemispheric fissure to the right by $7 \mathrm{~mm}$. Bilaterally the sulci and gyri were smoother compared to the previous CT study. Intra-parenchymally, left-frontally and cortically, an irregular small area could be seen, not clearly separated from the surrounding structures and with reduced density - up to $23 \mathrm{HE}$ relative to the surrounding brain parenchyma. Parasagittally, a new linear hypodesal zone was found, traceable along the entire axillary width up to $7 \mathrm{~mm}$. The swelling of the left upper eyelid was more pronounced and extended to the periorbital space. Inflammatory changes were not established infratentorially (Figure 6).

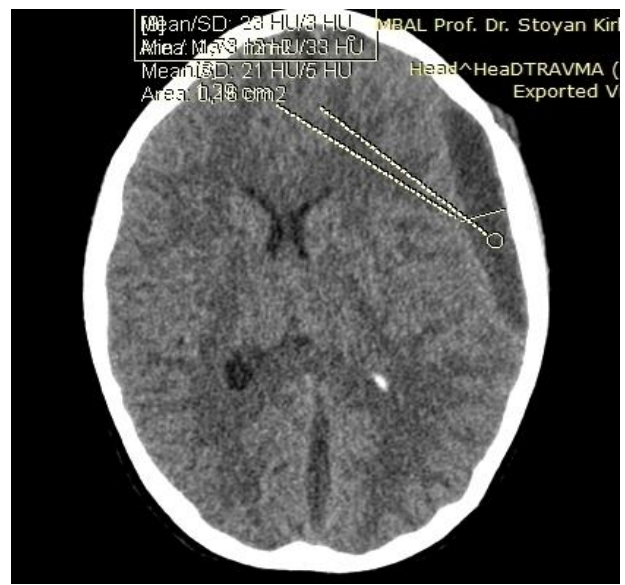

Figure 6. CT axial projection, left, subdural presence of hypodermic fluid collection and hypodermic changes bifrontal in progress
MITEV M., et al.

In conclusion: progress of cerebral oedema compared to the previous study; shaped progressive subdural abscess on the left, frontoparietally, compared to the previous scan; changes as with pronounced meningoencephalitis; some small lipoma on the left, frontally and parasagittally.

The subsequent head MRI confirmed stenosis of the subarachnoid spaces along the convexity of the two upper cerebral hemispheres. In the course of the meninges, bilaterally, frontally and on the left, frontotemporoparietally and parasagittally, the presence of pronounced high signal changes was detected. Intraparenchymally, cortically, bilaterally, frontally and more pronounced on the left, in the area of gyrus frontalis sinistra, gyrus precentralis sinistra et gyrus centralis sinistra, poorly delimited high-signal foci in $\mathrm{T} 2 \mathrm{w}$ sequence and low-signal foci in $\mathrm{T} 1 \mathrm{w}$ sequences were seen. On the left, subdurally, frontoparietally, a well-delimited high-signal region in the $\mathrm{T} 2 \mathrm{w}$ sequence and low-signal region in the $\mathrm{T} 1 \mathrm{w}$ sequence could be seen. In T2 FLAIR, the finding was with high signal changes in its peripheral part and low signal in the central area. It compressed the surrounding structures, slightly stenosed the body of the ipsilateral lateral ventricle and dislocated the interhemispheric fissure to $7 \mathrm{~mm}$ contralaterally on the right. Retrobulbar spaces were normal. The high signal changes were in the $\mathrm{T} 2 \mathrm{w}$ sequence, in the left upper eyelid and the periorbital space. Following the administration of contrast medium (Clariscan, $20 \mathrm{ml}$ ) intravenously, gadolinium chelates were found to be trapped in the described areas along the meninges, more pronounced on the left. The described subdural finding on the left indicated an accumulation of gadolinium chelates peripherally and no accumulation in the central area where diffusion and perfusion were not detected. The sellar and parasellar structures had normal dimensions and signal characteristics. The infratentorial cerebral parenchyma showed no pathological signal changes and no gadolinium chelates areas were seen. Paranasal cavities in the study volume had high-signal foci in $\mathrm{T} 2 \mathrm{w}$ sequence bilaterally, in the maxillary sinuses and ethmoidal cells were low-signal in the central part of the left maxillary sinus and the base of the right maxillary sinus in $\mathrm{T} 1$ and $\mathrm{T} 2$ FLAIR (Figure 7-10).

Conclusion: The MRI data described corresponded to changes as at 
meningoencephalitis in progress; an abscess was formed on the left, subdurally with the passage of the collection to the interhemispheric space; cerebral oedema; lipoma on the left, frontally and parasagittally; suppurative left-sided maxillary, ethmoidal sinusitis.

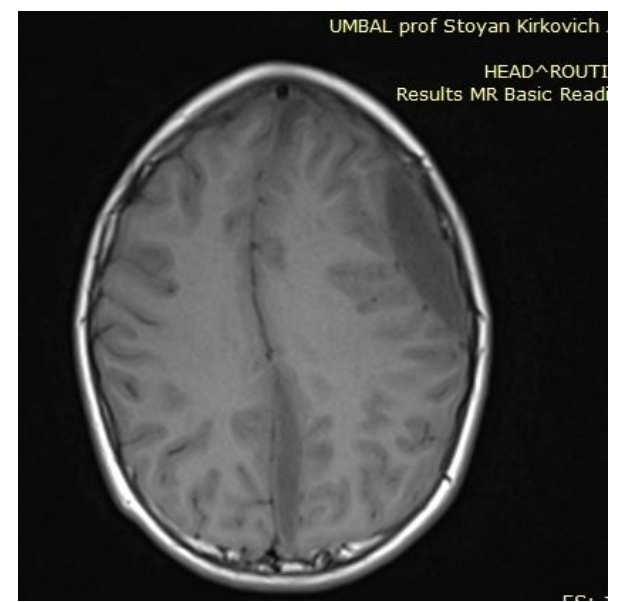

Figure 7. MRI T1 sequence, axial section. Low left, subdural and bilateral frontal changes in progress

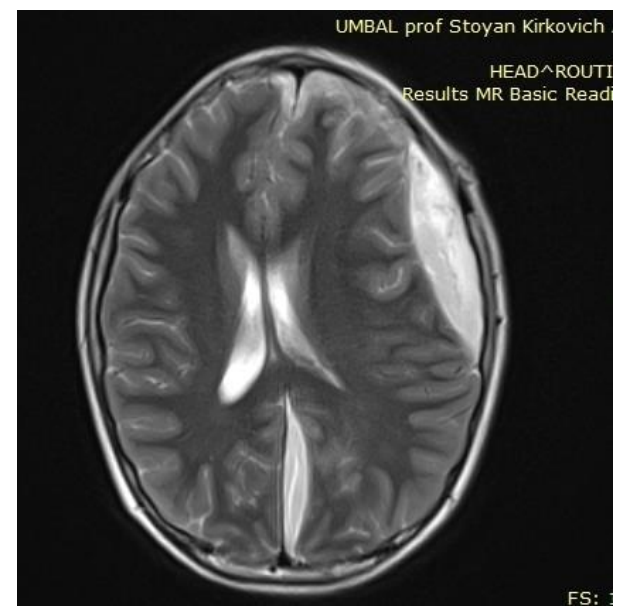

Figure 8. T2 FLAIR sequence, coronary projection. Low signal changes from left fluid, subdural, and high sensory changes of the cerebral sheaths and bifrontal cerebral parenchyma in progress

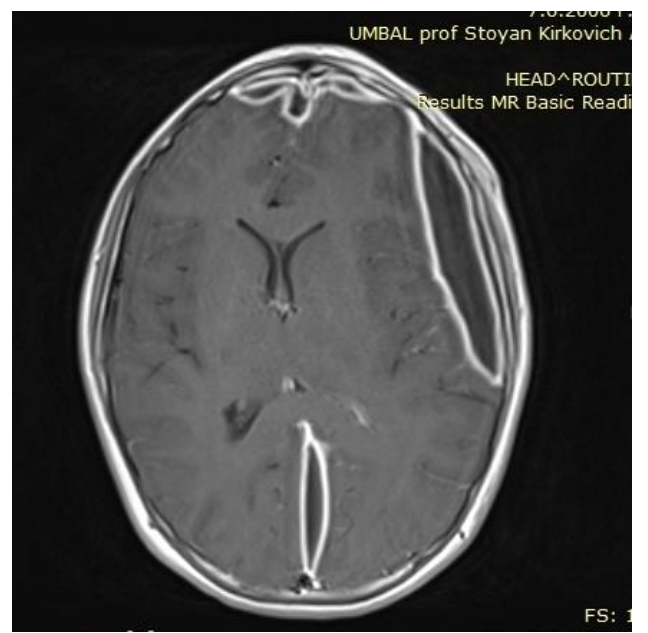

Figure 9. MRI T2 FLAIR, oxidation plane. Low-signal changes of fluid collection on the left, subdural and high-
MITEV M., et al. signal changes of cerebral sheaths and intra-parenchymal, bifrontal

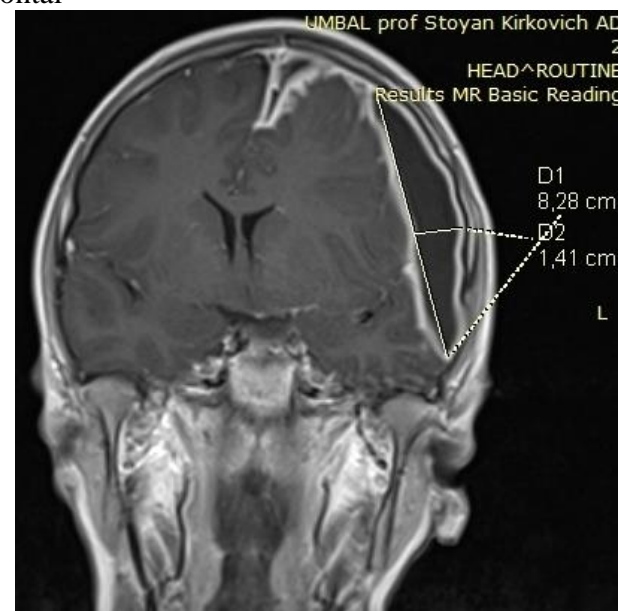

Figure 10. MRI T2 FLAIR, changes in coronary projection

\section{DISCUSSION}

Kastrup et al. (1), in cases of suspected bacterial meningitis, recommend immediate cranial CT before lumbar puncture and initiation of empirical antimicrobial treatment before CT. In the early phase of meningitis, the authors reported normal results of native CT. In later stages, persistent drowsiness and meningeal signs are considered as indications for a secondary CT. They state that MRT is not routinely required in cases of uncomplicated bacterial meningitis, but the visibility of the meninges on the pre-contrast $\mathrm{T} 1 \mathrm{w}$ sequences can be considered as a guide for tuberculous meningitis. In complex cases with convulsions and evolving focal signs, the authors cite MRI as being better than CT in demonstrating parenchymal lesions due to meningoencephalitis or vascular complications on FLAIR sequences. MRI is cited as a more sensitive imaging method that shows a high periventricular signal on FLAIR images, etc. According to Suresh et al. (3), congenital neuroectodermal defects are associated with meningitis that does not respond to conventional antibiotic therapy, recurrent bacterial meningitis, or meningitis due to an organism that is not usually the cause of the disease. They point out that mid-thoracic dermoid and epidermoid cysts are rare and are therefore easily overlooked. A case of a 13month-old boy with meningitis who did not respond to antimicrobial therapy is reported. Subsequently, they identify an intraspinal abscess, an infection due to Staphylococcus epidermidis, and a mid-thoracic dermoid cyst. They emphasize the need for clinicians to have a high index of suspicion and to perform a careful physical examination when antibiotic 
MITEV M., et al.

treatment fails in patients with meningitis. They also provide detailed anatomy of a midthoracic dermoid cyst and illustrate the usefulness of magnetic resonance imaging. Younis et al. (2) performed a retrospective analysis of all patients diagnosed with sinusitis with complications. They point out that meningitis is the most common intracranial complication. Intracranial complications were reported in 39 of 82 patients, and orbital complications in 43 patients. Twenty-one of thirty-nine intracranial complications is classified as meningitis. The most common CT result detected in 54\% of adult patients is identified as sphenoid sinusitis. In all AIDS patients (6 of 21), cryptococcal meningitis is detected, and in patients without AIDS, Streptococcus pneumoniae (10 of 21) is reported as the most common. According to the authors, the introduction of high-resolution $\mathrm{CT}$, magnetic resonance imaging and the presence of broad-spectrum antibiotics significantly improve disease management. Morita and Ishihara (4) present acute central nervous system infections with an emphasis on bacterial meningitis and herpes simplex encephalitis. In $75 \%$ of the cases of meningitis in Japan, they report pneumonic meningitis. The aetiology of acute encephalitis is often unknown, according to the authors. Herpes simplex is cited as one of the main causes with extremely poor prognosis without treatment. Timothy and Rodger (5) describe a case of meningoencephalitis after endoscopic dacryocystorhinostomy in an 81-year-old man with unilateral nasolacrimal duct obstruction, who underwent routine endonasal dacryocystorhinostomy, and after two days he developed severe headache and rigour. Subsequent CT and MRT revealed a defect at the base of the right anterior cranial fossa, which was probably caused during surgery, and meningoencephalitis of the curvature of the adjacent frontal lobe. After intravenous antibiotic treatment, the patient is fully recovered without any lasting effects. According to the authors, this is the first report of meningoencephalitis after endonasal dacryocystorhinostomy. This complication, according to the authors, should be considered in any patient with febrile septic presentation or headache after endonasal dacryocystorhinostomy.

Pekova et al. (6) reported a case of a patient (29-year-old man) with Listeria monocytogenes. They reported $L$. monicytogenes as the third most frequent cause of bacterial meningitis. After MRI (Magnetic Resonance Imaging), the author's appeared massive cerebral oedema. The imagings of the method of Computer tomography with contrast showed persistent cerebral oedema. By the technique of CSF's data, they confirmed them, etc. Listeria monocytogenes is reported as one of the causes of infection in the Central Nerves System (CNS), inconjunction with Streptococcus pneumoniae, Haemophilus influenzae and Neisseria menintitidis $(6,7)$. Pekova et al. (8) determined Serogroup B meningococcus (Neisseria meningitidis) as uncommon in Bulgaria.

Hughes et al. (10) reported MRI as extremely useful for detecting and monitoring the complications of meningitis.

According to this study, CT and MRI findings, as a result of the dynamic follow-up, showed initially only acute purulent left maxillary sinusitis, chronic right maxillary sinusitis and conjunctivitis with the rapid and dynamic development of the disease. The control CT after two days showed dynamics in the imaging CT finding with the appearance of changes in the type of brain oedema and development of pansinusitis. The consequent follow-up with CT showed rapid dynamics of changes in imaging CT and MRI studies, correlating with changes such as inflammation of the meninges and brain parenchyma, more pronounced on the left. The extension of the diagnostic algorithm with MRI, with the administration of intravenous contrast medium, clearly demonstrated changes characteristic of meningoencephalitis with a clearly formed subdural abscess on the left, frontoparietally, pansinusitis and conjunctivitis with periorbital oedema. The follow-up of the algorithm of changes over time led to the development of secondary meningoencephalitis, but most likely to be concomitant with primary purulent maxillary sinusitis with a transition to meningoencephalitis and brain abscess.

\section{CONCLUSION}

In this case, imaging methods clearly and accurately demonstrated the dynamics and assisted in making the right diagnosis, which would help to conduct the correct treatment and behavior in such patients with rapidly, dynamically developing and unspecified conditions. 


\section{REFERENCES}

1. Kastrup O, Wanke I,Maschke M. Neuroimaging of Infections. NeuroRx, 2(2):324-332, 2005. doi: 10.1602/neurorx.2.2.324

2. Younis R T, Anand V K,Childress C. Sinusitis Complicated by Meningitis: Current Management. Laryngoscope, 111(8):13381342,2001. https://doi.org/10.1 097/00005537-200108000-00006

3. Suresh G. Gurbani, Cheng T. Cho, Kyo R. Lee.Staphylococcus epidermidis Meningitis and an Intraspinal Abscess Associated with a Midthoracic Dermal Sinus Tract. Clinical Infectious Diseases, 19(6):1138-1140, 1994. https://doi.org/10.1093/clinids/19.6.1 138

4. Morita A, Ishihara M. Acute Infections of the Central Nervous System: Focus on Bacterial Meningitis and Herpes Simplex Encephalitis. Neurocritical Care, 187-194, 2019. DOI: https://doi.org/10.1007/978981-13-7272-8_15

5. Timothy Ch, Rodger D. Meningoencephalitis Following Endoscopic Dacryocystorhinostomy.Ophthalmic Plastic \& Reconstructive Surgery, 35(2):e47-e49, 2019.

doi: 10.1097/IOP.0000000000001324
MITEV M., et al.

6. Pekova L, Parusheva P, Rachkova K, Naydenov K, Tsoneva V, Mitev M. Listeria monocytogenes Meningoencephalitis in Immunocompetent Patient - Clinical Course and Outcome. Ann Clin Lab Res, 5(3):191, 2017. DOI: $10.21767 / 2386-5180.1000191$

7. Brouwer M C, Van De Beek D, Heckenberg S G, Spanjaard L, De Gans J. Community-acquired Listeria monocytogenes meningitis in adults. Clin Infect Dis, 43:1233-1238, 2006. DOI: $10.1086 / 508462$

8. Pekova L, Parousheva P, Lazarova G, Ananiev J. Two Cases of Meningococcal Sepsis Caused By Neisseria Meningitidis Serogroup B. J Clin Anal Med, 1-3, 2015. DOI: $10.4328 / \mathrm{JCAM} .4130$.

9. Dzupova O,Rozsypal H,Smiskova D,Benes J. Listeria monocytogenes Meningitis in Adults: The Czech Republic Experience. BioMed Research International, 1-4, 2013, http://dx.doi.org/10.1155/2013/846186

10. Hughes D C, Raghavan A, Mordekar S R, Griffiths P D, Connolly D J A. Role of imaging in the diagnosis of acute bacterial meningitis and its complications. $P M J$, 86(1018),2010.http://dx.doi.org/10.1136/pgmj. 2010.097022 